\title{
LAX TENSORS, KILLING TENSORS AND GEOMETRIC DUALITY
}

\author{
Dumitru Baleanuf \\ Middle East Technical University , Physics Department-06531 Ankara,Turkey \\ and \\ Institute of Space Sciences, P.O.BOX, MG-23, R 76900,Magurele-Bucharest, \\ Romania \\ and \\ Ayşe (Kalkanlı) Karasuf \\ Middle East Technical University, Physics Department-06531, \\ Ankara, Turkey
}

\begin{abstract}
The solution of the Lax tensor equations in the case $L_{\alpha \beta \gamma}=-L_{\beta \alpha \gamma}$ was analyzed. The Lax tensors on the dual metrics were investigated. We classified all two dimensional metrics having the symmetric Lax tensor $L_{\alpha \beta \gamma}$. The Lax tensors of the flat space, Rindler system and its dual were found.
\end{abstract}

\section{Introduction}

Killing tensors are indispensable tools in the quest for exact solutions in many branches of general relativity as well as classical mechanics [1]. Killing tensors are important for solving the equations of motion in particular space-times. The notable example here is the Kerr metric which admits a second rank Killing tensor [1].Killing tensors give rise to new exact solutions in perfect fluid Bianchi and Katowski-Sachs cosmologies as well in inflationary models with a scalar field sources [2]. Recently Killing tensors of third rank in $(1+1)$ dimensional geometry were investigated and classified [3].Even more recently the Killing tensors of order two associated with orthogonal separable coordinates for the Klein-Gordon equation in flat $2+1$ dimensional space-time were considered as metrics 《4. In a geometrical setting, symmetries are connected with isometries associated with Killing vectors, and more generally, Killing tensors on the

\footnotetext{
${ }^{1}$ E-mail: baleanu@venus.nipne.ro, dumitru@newton.physics.metu.edu.tr

${ }^{2}$ E-mail:akarasu@metu.edu.tr
} 
configuration space of the system.An example is the motion of a point particle in a space with isometries, which is a physicist's way of studying the geodesic structure of a manifold [5]. We recall that $K_{\alpha \beta}$ is a Killing tensor if and only if , for any geodesic motion of a test particle with a world velocity $p^{\alpha}$, the scalar $K_{\alpha \beta} p^{\alpha} p^{\beta}$ is a constant of motion [6]. The Jacobi's geometrical model of dynamical systems with a finite number of degrees of freedom was investigated by many authors (see for example Refs. [7, 8]). The essential conclusion was that :the paths of the motions of a dynamical system in the configuration space are identical with the geodesics of the Riemannian manifold obtained by providing the configuration space with the metric given by

$$
d s^{2}=g_{i j} d q_{i} d q_{j}=2(E-V) a_{i j} d q_{i} d q_{j} .
$$

We mention here that $T=\frac{1}{2} a_{i j} \dot{q}_{i} \dot{q}_{j}$ (the dot signifying derivation with respect to time and $a_{i j}$ are functions of the q's), $\mathrm{V}$ is a function of the q's only and $T+V=E$. In [9] it was pointed out that a single Lax tensor may generate an infinite number of tensors of varying ranks.It is very well known that the most general constant on a geodesic motion is of the form

$$
K=K_{0}+\chi_{\mu} p^{\mu}+K_{\mu \nu} p^{\mu} p^{\nu}+K_{\mu \nu \lambda} p^{\mu} p^{\nu} p^{\lambda}+\cdots
$$

where $K_{0}$ is a constant of motion on the geodesic , $\chi_{\mu}$ is a Killing vector and $K_{\mu_{1} \cdots \mu_{n}}$ is a Killing tensor of order $\mathrm{n}$. The important point is that if we are using Jacobi's geometrical model a natural way to produce Killing tensors is to consider the elements of the Lax matrix $L_{\alpha \beta}$ [10, 11] as $L_{\alpha \beta}=L_{\alpha \beta}^{\gamma} p_{\gamma}+C_{\alpha \beta}$ [12]. Here $C_{\alpha \beta}$ is a matrix having the elements satisfying the following relations $\operatorname{tr}\left(C_{\alpha \beta}\right)=K_{0}, L_{\alpha \beta}^{\alpha}=\chi_{\beta}, K_{\alpha \beta}=L_{\nu \alpha}^{\mu} L_{\mu \beta}^{\nu}$ and so on. Open three dimensional Toda's case analyzed in [9] is a special and very interesting case because the Lax tensor generates a Killing tensor of order two which is equal to the metric tensor.We know that in this case Killing tensor of order two is called trivial (see for more details 133). Recently the geometric duality between a metric $g^{\mu \nu}$ and its non-degenerate Killing tensor $K^{\mu \nu}$ and the structural equations of a Killing tensor of order two were analyzed in [14, 15]. An interesting example arises when the manifold admits Killing-Yano tensors [16] because they generate Killing tensors.In addition we know that any manifold having constant curvature admits Killing-Yano tensors and then it admits Killing tensors.

For these reasons the Lax tensor equations on a given manifold and its dual are interesting to investigate.

The plan of this paper is as follows:

In Section 2 the Lax pair tensors are investigated.In Section 3 the geometric duality is presented and the Lax tensors on the dual manifolds are analyzed.In Section 4 the examples are presented.Section 5 contains our comments and remarks. 


\section{Lax pair tensors}

Let us consider a Riemannian or pseudo-Riemannian geometry with the metric

$$
d s^{2}=g_{\mu \nu} d q^{\mu} d q^{\nu}
$$

The geodesic equation can be represented by the Hamiltonian

$$
H=\frac{1}{2} g^{\mu \nu} p_{\mu} p_{\nu}
$$

together with the natural Poisson bracket on the cotangent bundle. The geodesic system has the form

$$
\dot{q}^{\alpha}=g^{\alpha \mu} p_{\mu}, \dot{p}_{\alpha}=\Gamma_{\alpha}^{\mu \nu} p_{\mu} p_{\nu}
$$

The complete integrability of this system can be shown with the help of a pair of matrices L and A with entries defined on the phase space and satisfying the Lax pair equation [10, 11].

$$
\dot{L}=\{L, H\}=[L, A]
$$

It follows from (6) that the quantities $I_{k}=\frac{1}{k} \operatorname{Tr} L^{k}$ are all constants of motion. If in addition they commute with each other $\left\{I_{k}, I_{j}\right\}=0$ then it is possible to integrate the system completely at least in principle. We know that Lax pair equation is invariant under a transformation of the form

$$
\tilde{U}=U L U^{-1}, \tilde{A}=U A U^{-1}-\dot{U} U^{-1}
$$

We see that $\mathrm{L}$ transforms as a tensor while A transforms as a connection. Typically,the Lax matrices are linear in the momenta and in the geometric setting that may also be assumed to be homogeneous. This motivates the introduction of two third rank geometrical objects $L_{\beta}^{\alpha \gamma}$ and $A_{\beta}^{\alpha \gamma}$ such that the Lax matrices can be written as

$$
L=\left(L_{\beta}^{\alpha}\right)=\left(L_{\beta}^{\alpha \mu} p_{\mu}\right), A=\left(A_{\beta}^{\alpha}\right)=\left(A_{\beta}^{\alpha \mu} p_{\mu}\right) .
$$

We will refer to $L_{\beta}^{\alpha \gamma}$ and $A_{\beta}^{\alpha \gamma}$ as the Lax tensor and the Lax connection , respectively.Defining

$$
B=\left(B_{\beta}^{\alpha}\right)=\left(B_{\beta}^{\alpha \mu} p_{\mu}\right)=A-\Gamma
$$

where $\Gamma=\left(\Gamma_{\beta}^{\alpha}\right)=\left(\Gamma_{\beta}^{\alpha \mu} p_{\mu}\right)$ is the Levi-Civita connection with respect to $g_{\alpha \beta}$, it then follows that the Lax pair equation takes the covariant form. Let us suppose that a manifold $g_{\mu \nu}$ admits a Lax pair tensors $L_{\alpha \beta \gamma}, A_{\alpha \beta \gamma}$ in such a way that

$$
L_{\alpha \beta \gamma ; \delta}+L_{\alpha \beta \delta ; \gamma}=L_{\alpha \mu(\gamma} B_{|\beta| \delta)}^{\mu}-B_{\alpha \mu(\gamma} L_{|\beta| \delta)}^{\mu} .
$$


Here the parenthesis denotes the full symmetrization. We know that a Killing tensor of order $\mathrm{n}$ is a symmetric tensor $K_{\mu_{1} \cdots \mu_{n}}$ which satisfies the following relation:

$$
D_{(\lambda} K_{\left.\mu_{1} \cdots \mu_{n}\right)}=0
$$

where $D_{\mu}$ denote covariant derivative. and Using (10), in the case when $L_{\alpha \beta \gamma}$ has only symmetric part, we find immediately that $L_{(\alpha \beta \gamma ; \delta)}=0$ for $B_{\beta \gamma}^{\alpha}=0$ . Then it is a Killing tensor of order three. Any solution of (10) generates an infinite number of Killing tensors on a given manifold.Of course not all Killing tensors generated by Lax tensors are independent and some of them are trivial Killing tensors [13. Another important observation is that in the case when we have $g_{\alpha \beta}=L_{\nu \alpha}^{\mu} L_{\mu \beta}^{\nu}$ we can identify the invariant $I_{2}$ with the geodesic Hamiltonian.

Let us suppose that the manifold admits a Killing tensors $K_{\alpha \beta}$ and define a three dimensional tensor as

$$
L_{\alpha \beta \gamma}=K_{\beta \gamma ; \alpha}-K_{\alpha \gamma ; \beta} .
$$

We conclude immediately that it has the symmetries

$$
L_{\alpha \beta \gamma}=L_{[\alpha \beta] \gamma}, L_{[\alpha \beta \gamma]}=0,
$$

where square brackets denote the anti-symmetrization. After an appropriate grouping of terms and use of the symmetries of the Riemann tensor $R_{\alpha \beta \gamma \delta}$ we obtain

$$
L_{\alpha \beta(\gamma ; \delta)}=-2 R_{\alpha \beta \mu(\gamma} K_{\delta)}^{\mu}-2 K_{[\alpha}^{\mu} R_{\beta] \mu(\gamma \delta) \mu} .
$$

We are interested now to investigate if (12) satisfies (10). In other words our problem is to find a tensor $B_{\alpha \beta \gamma}$ in such a way that (10) is satisfied.Using (12) and (10) we conclude that $B_{\alpha \beta \gamma}=-B_{\beta \alpha \gamma}$. Let us denote $V_{\alpha \beta \gamma}$ as $V_{\alpha \beta \gamma}=L_{[\alpha \beta] \gamma}$. Taking into account (10) and (14) we find that

$$
V_{\alpha \mu(\gamma} B_{|\beta| \delta)}^{\mu}-V_{\beta \mu(\gamma} B_{|\alpha| \delta)}^{\mu}=R_{\alpha \beta \mu(\gamma} K_{\delta)}^{\mu}+K_{[\alpha}^{\mu} R_{\beta] \mu(\gamma \delta) \mu} .
$$

Solving (15) we can determine $B_{\beta \gamma}^{\alpha}$.

Using definition of a Killing tensor of order two and (12) we get

$$
K_{\beta \gamma ; \alpha}=\frac{2}{3} L_{\alpha(\beta \gamma)}
$$

Conversely , (16) and the conditions $L_{\alpha \beta \gamma}=-L_{\beta \alpha \gamma}, L_{[\alpha \beta \gamma]}=0$ imply (12) and that $K_{\alpha \beta}$ is a Killing tensor.

Another interesting case is when $L_{\alpha \beta \gamma}=V_{\alpha \beta \gamma}$ and in addition we suppose that $B_{\alpha \beta \gamma}=L_{\alpha \beta \gamma}$. For this case the Lax equations become

$$
V_{\alpha \beta(\gamma ; \delta)}=0,
$$

and we see that (17) looks like Killing-Yano equations. 


\section{Geometric Duality}

Let us suppose that the metric $g_{\mu \nu}$ admits a Killing tensor field $K_{\mu \nu}$.

From the covariant components $K_{\mu \nu}$ of the Killing tensor one can construct a constant of motion $K=\frac{1}{2} K_{\mu \nu} p^{\mu} p^{\nu}$. It can be verified that $\{H, K\}=0$. The formal similarity between the constants of motion $\mathrm{H}$ and $\mathrm{K}$, and the symmetrical nature of the condition implying the existence of the Killing tensor amount to a reciprocal relation between two different models:the model with Hamiltonian $\mathrm{H}$ and constant of motion $\mathrm{K}$, and a model with constant of motion $\mathrm{H}$ and Hamiltonian K. The relation between the two models has a geometrical interpretation: it implies that if $K_{\mu \nu}$ are the contravariant components of a Killing tensor with respect to the metric $g_{\mu \nu}$, then $g_{\mu \nu}$ must represent a Killing tensor with respect to the metric defined by $K_{\mu \nu}$. When $K_{\mu \nu}$ has an inverse we interpret it as the metric of another space and we can define the associated Riemann-Christoffel connection $\hat{\Gamma}_{\mu \nu}^{\lambda}$ as usual through the metric postulate $\hat{D}_{\lambda} K_{\mu \nu}=0$. Here $\hat{D}$ represents the covariant derivative with respect to $K_{\mu \nu}$. This reciprocal relation between the metric structure of pairs of spaces constitutes a duality relation: performing the operation of mapping a Killing tensor to a metric twice leads back to the original theory.

The relation between connections $\hat{\Gamma}_{\alpha \beta}^{\sigma}$ and $\Gamma_{\alpha \beta}^{\sigma}$ is [15]

$$
\hat{\Gamma}_{\alpha \beta}^{\mu}=\Gamma_{\alpha \beta}^{\mu}-K^{\mu \delta} D_{\delta} K_{\alpha \beta} .
$$

In the case when the tensor $B_{\beta \delta}^{\alpha}$ is symmetric in the lower indices and has the form

$$
B_{\beta \delta}^{\alpha}=K^{\alpha \omega} D_{\omega} K_{\beta \delta}
$$

then $(\sqrt{10})$ becomes

$$
\hat{L}_{\alpha \beta \gamma ; \delta}+\hat{L}_{\alpha \beta \delta ; \gamma}=0 .
$$

Here comma represents the covariant derivative in the dual space. We are interested now to investigate when the original space and its dual admit the same Lax tensors.

\section{Proposition}

The manifold and its dual have the same Lax tensors iff

$$
\begin{aligned}
2\left(K^{\sigma \omega} D_{\omega} K_{\gamma \delta}\right) L_{\alpha \beta \sigma} & +\left(K^{\sigma \omega} D_{\omega} K_{\alpha \delta}\right) L_{\sigma \beta \gamma}+\left(K^{\sigma \omega} D_{\omega} K_{\alpha \gamma}\right) L_{\sigma \beta \delta}+ \\
\left(K^{\sigma \omega} D_{\omega} K_{\beta \delta}\right) L_{\alpha \sigma \gamma} & +\left(K^{\sigma \omega} D_{\omega} K_{\beta \gamma}\right) L_{\alpha \sigma \delta}=0 .
\end{aligned}
$$

Proof.

Let us consider $L_{\alpha \beta \gamma}$ the Lax tensor satisfies

$$
L_{\alpha \beta(\gamma ; \delta)}=0
$$


and $\hat{L}_{\alpha \beta \gamma}$ be the dual Lax tensor. Using (18) the corresponding dual Lax equations are

$$
\begin{array}{cl}
D_{\delta} \hat{L}_{\alpha \beta \gamma} & +D_{\gamma} \hat{L}_{\alpha \beta \delta}+2\left(K^{\sigma \omega} D_{\omega} K_{\gamma \delta}\right) \hat{L}_{\alpha \beta \sigma}+\left(K^{\sigma \omega} D_{\omega} K_{\alpha \delta}\right) \hat{L}_{\sigma \beta \gamma}+ \\
\left(K^{\sigma \omega} D_{\omega} K_{\alpha \gamma}\right) \hat{L}_{\sigma \beta \delta} & +\left(K^{\sigma \omega} D_{\omega} K_{\beta \delta}\right) \hat{L}_{\alpha \sigma \gamma}+\left(K^{\sigma \omega} D_{\omega} K_{\beta \gamma}\right) \hat{L}_{\alpha \sigma \delta}=0
\end{array}
$$

Let us suppose that $\hat{L}_{\alpha \beta \gamma}=L_{\alpha \beta \gamma}$, then using (22) and (23) we obtain (21) Conversely if we suppose that (21) holds, then from (23) we can deduce immediately that $L_{\alpha \beta \gamma}=\hat{L}_{\alpha \beta \gamma}$. q.e.d.

\section{Examples}

In this section we will present some examples when the equations (10) admit solutions.

A.

Let us consider the n-dimensional Euclidean space and first let us investigate the Lax equations corresponding to $B_{\beta \gamma}^{\alpha}=0$. Then (10) becomes

$$
\frac{\partial L_{\alpha \beta \gamma}}{\partial x^{\delta}}+\frac{\partial L_{\alpha \beta \delta}}{\partial x^{\gamma}}=0
$$

The solution of this equation has the form

$$
L_{\alpha \beta \gamma}=T_{\alpha \beta \gamma \sigma} x^{\sigma}+V_{\alpha \beta \gamma}
$$

where $T_{\alpha \beta \gamma \sigma}$ and $V_{\alpha \beta \gamma}$ are constant tensors and in addition $T_{\alpha \beta \gamma \sigma}=-T_{\alpha \beta \sigma \gamma}$.

B.

Let us consider now the two dimensional metric

$$
d s^{2}=f(u, v) d u^{2}+g(u, v) d v^{2}
$$

We are interested to investigate the Lax tensors when $L_{\alpha \beta \gamma}$ is symmetric and $A_{\alpha \beta \gamma}=\Gamma_{\alpha \beta \gamma}$. The non-vanishing Christoffel symbols of (26) are

$$
\begin{aligned}
& \Gamma_{11}^{1}=\frac{\frac{\partial f}{\partial u}}{2 f}, \Gamma_{11}^{2}=\frac{-\frac{\partial f}{\partial v}}{2 g}, \Gamma_{21}^{1}=\Gamma_{12}^{1}=\frac{\frac{\partial f}{\partial v}}{2 f}, \\
& \Gamma_{22}^{2}=\frac{-\frac{\partial g}{\partial u}}{2 f}, \Gamma_{22}^{2}=\frac{\frac{\partial g}{\partial v}}{2 g}, \Gamma_{21}^{1}=\Gamma_{12}^{2}=\frac{\frac{\partial g}{\partial u}}{2 g} .
\end{aligned}
$$

$L_{\alpha \beta \gamma}$ has four independent components $L_{111}, L_{112}, L_{122}, L_{222}$ and the independent Lax equations are

$$
\begin{aligned}
& L_{11(1 ; u)}=0 \quad, L_{11(1 ; v)}=0, L_{11(2 ; u)}=0, L_{11(2 ; v)}=0 \\
& L_{12(2 ; u)}=0 \quad, L_{12(2 ; v)}=0, L_{22(2 ; u)}=0, L_{22(2 ; v)}=0 .
\end{aligned}
$$


We found after some calculations that if the scalar curvature of the manifold corresponding to (26) is 0 then the system (28) is integrable.

Let us consider now the Rindler system. The Rindler system [17] is conventionally denoted by $\tau$ and $\mathrm{r}$

$$
t=r \sinh \tau, x=r \cos \tau, 0<r<\infty,-\infty<\tau<\infty
$$

with coordinates curves (timelike hyperbolas and spacelike straight lines) given by

$$
x^{2}-t^{2}=r^{2}, \frac{t}{x}=\tanh \tau
$$

the metric

$$
d s^{2}=r^{2} d \tau^{2}-d r^{2}
$$

and the associated Killing tensor

$$
k^{i k}=\left(\begin{array}{cc}
1-\frac{c}{r^{2}} & 0 \\
0 & c
\end{array}\right) .
$$

Here $\mathrm{c}$ is a constant. The non-zero Christoffel symbols are $\Gamma_{11}^{2}=r, \Gamma_{12}^{1}=\frac{1}{r}$. Solving (28) we found the solution of the Lax equations having the form

$$
\begin{aligned}
& L_{122}=\left(C_{1} \mathrm{e}^{-\tau}+C_{2} \mathrm{e}^{3 \tau}\right) r, L_{112}=\left(C_{1} \mathrm{e}^{-\tau}+C_{2} \mathrm{e}^{3 \tau}\right) r^{2}, \\
& L_{111}=-\left(3 C_{1} \mathrm{e}^{-\tau}-C_{2} \mathrm{e}^{3 \tau}\right) r^{3}, L_{222}=-3 C_{1} \mathrm{e}^{-\tau}+C_{2} \mathrm{e}^{3 \tau},
\end{aligned}
$$

where $C_{1}, C_{2}$ are constants.

The next step is to find a solution of the form (12) corresponding to the Rindler system. Using (12) and (32) we found immediately the solution having the form

$$
L_{121}=-\frac{r^{3}\left(r^{2}-3 c\right)^{2}}{c\left(r^{2}-c\right)^{2}}, L_{122}=0 .
$$

Let us consider now the tensor

$$
K^{\mu \nu}=g^{\mu \lambda} g^{\nu \delta} K_{\lambda \delta}
$$

and a connection defined as in (18). Using (18) and taking into account (35)and (32) we found a new metric having the non-zero components

$$
d \hat{s}^{2}=r^{2} d \tau^{2}+\frac{c\left(r^{2}-c\right)^{2}}{\left(2 r^{2}+r^{4} c-2 r^{2} c^{2}+c^{3}\right)} d r^{2} .
$$

The scalar curvature corresponding to (36) is $R=\frac{4\left(r^{2}+c\right)}{c\left(-r^{2}+c\right)^{3}}$, then this metric has no symmetric Lax tensors. 


\section{Concluding remarks}

In this paper we investigated the Lax equations on a given manifold and its dual. When a manifold admits a Killing tensor $K_{\mu \nu}$ we constructed a tensor $L_{\alpha \beta \gamma}$ as $L_{\alpha \beta \gamma}=K_{\beta \gamma ; \alpha}-K_{\alpha \gamma ; \beta}$ and found the conditions when it is a Lax tensor.In this case $L_{\alpha \beta \gamma}$ is antisymmetric in the first two indices and $B_{\alpha \beta \gamma}$ should have the same property. If in addition we suppose that $B_{\beta \alpha \gamma}=L_{\alpha \beta \gamma}$ we found that (10) has the simple form $L_{\alpha \beta(\gamma ; \delta)}=0$. We found the conditions when the manifold and its dual have the same Lax tensors. For the two dimensional manifolds we found that the symmetric Lax tensors exist if the scalar curvature is zero. The solution of the Lax equations for the flat space case, the Rindler system and its dual manifold were found.

Finding the Lax tensors on the manifolds which admits Killing-Yano tensors is an interesting problem and it requires further investigation.

\section{Acknowledgements}

One of the authors (D.B.) would like to thank Ashok Das for helpful discussions. He also would like to thank TUBITAK and NATO for financial support and METU for the hospitality during his working stage at the Department

of Physics. This work is partially supported by the Scientific and Technical Research Council of Turkey.

\section{References}

[1] G.W. Gibbons, R.H.Rietdijk and J.W.van Holten, Nucl.Phys. B 404, 42 (1993).

[2] K.Rosquist and C.Uggla, J.Math.Phys. 32, 3412 (1991).

[3] M. Karlovini and K. Rosquist, preprint gr-qc/9807051.

[4] F.Hinterleitner, Annals of Physics 271,23 (1999).

[5] R.H.Rietdijk and J.W.van Holten, J.Geom.Phys. 11, 559 (1993).

[6] L.P.Eisenhart, Riemannian Geometry (Princeton U.P., Princeton, N.J.) (1966).

[7] H.E.Rauch, Geodesics and curvature in differential geometry in the large, (Yeshiva University, New York, 1959).

[8] K.Rosquist, in The Seventh Marcel Grossmann Meeting.On Recent Developmnets in Theoretical and Experimental General Relativity, Gravitation 
and Relativistic Field Theories,edited by.R.T.Jantzen and G.M.Keiser (World Scientific, Singapore), 1997 vol.1, p.379.

[9] K. Rosquist and M.Goliath, Gen.Rel.Grav. 30 ,1521 (1998).

[10] P.D.Lax, Comm.Pure.Appl.Math. 21, 467 (1968).

[11] A.M.Perelomov, Integrable systems of classical mechanics and Lie algebra, I.Birkhauser, 1990.

[12] M.Goliath,M.Karlovini,Kjell Rosquist, solv-int/9810011.

[13] D.Kramer ,H.Stephani, E.Herlt and M.Mac Callum Exact Solutions of Einstein Field Equations, Cambridge University Press, 1980.

[14] R.H.Rietdjik, J.W. van Holten Nucl.Phys. B 472 ,472 (1996).

[15] D.Baleanu and S.Codoban , Gen. Rel. and Grav. 31,497 (1999), D.Baleanu, Preprint JINR E5-98-188, D.Baleanu,in Proceedings of XIth Intl.Conf.Problems of Quantum Field Theory , Dubna, Russia, 13-17 July, p.66 (Publ.Dubna 1999, Ed. B.M.Barbashov, G.V.Efimov and A.V.Efremov), D.Baleanu, to be published in Supplemento ai Rendiconti del Circolo Matematico di Palermo.

[16] K.Yano, Ann. Math., 55, 328 (1952).

[17] F.Hinterleitner, Acta. Phys.Slovaca 47 , 157 (1997). 